\title{
Oxaliplatin-Induced Peripheral Neuropathy Can Be Minimized By Pressurized Regional Intravascular Delivery In An Orthotopic Murine Pancreatic Cancer Model.
}

Jayanth Surya Narayanan Shankara Narayanan

University of California San Diego

Katie Frizzi

University of California San Diego

Suna Erdem

University of California San Diego

Partha Ray

University of California San Diego

David Jaroch

TriSalus $^{\text {TM }}$ Life Sciences, Inc

Bryan Cox

TriSalus $^{\text {TM }}$ Life Sciences, Inc

Steven Katz

TriSalus ${ }^{\text {TM }}$ Life Sciences, Inc

Diego Vicente

Uniformed Services University Health System

Rebekah R. White ( $\square$ rewhite@health.ucsd.edu )

University of California San Diego

Short Report

Keywords: pancreatic, oxaliplatin, tumor, neuropathy

Posted Date: January 6th, 2022

DOI: https://doi.org/10.21203/rs.3.rs-1226505/v1

License: () (1) This work is licensed under a Creative Commons Attribution 4.0 International License.

Read Full License 


\section{Abstract}

Purpose: There is a great need to reduce the toxicity of chemotherapy used in the management of pancreatic ductal adenocarcinoma (PDAC). Here we explore if regional pressurized delivery of oxaliplatin can minimize peripheral neuropathy in mice.

Methods: We used an orthotopic PDAC mouse model and delivered a single dose of oxaliplatin through the portal vein using a pressure-enabled system (pancreatic retrograde venous infusion, PRVI). We analyzed the effects of PRVI on tumor burden and peripheral neuropathy using histopathological and functional assays.

Results: Tumor weights in mice treated with $2 \mathrm{mg} / \mathrm{Kg}$ oxaliplatin using PRVI were significantly lower than in mice treated with the same dose systemically. This resulted in reduced peripheral neuropathy signatures in PRVI mice compared to the $20 \mathrm{mg} / \mathrm{Kg}$ systemic dose required to achieve similar tumor control.

Conclusion: Regional delivery of highly cytotoxic agents using PRVI can reduce the therapeutic dose of these drugs, thereby lowering toxic side effects.

\section{Introduction}

Pancreatic Ductal Adenocarcinoma (PDAC) is one of the most aggressive major cancers and usually presents with locally advanced and/or metastatic disease [1]. Consensus guidelines are that patients with good performance status should receive systemic chemotherapy as their first line of therapy [2]. The current "optimal" regimens are either gemcitabine with nab-paclitaxel or FOLFIRINOX (folinic acid, 5-FU, irinotecan, and oxaliplatin) [3]. The highest objective response rates have been seen in studies of FOLFIRINOX but are in the range of only $20-40 \%$ [4], and this regimen is associated with high toxicity [5]. FOLFIRINOX is notable for its high incidence of grade 3 to 4 adverse events including neutropenia (45.7\% vs $21.0 \%$ ), febrile neutropenia ( $5.4 \%$ vs $1.2 \%$ ), thrombocytopenia ( $9.1 \%$ vs $3.6 \%$ ), diarrhea (12.7\% vs $1.8 \%$ ), and peripheral neuropathy ( $9 \%$ vs $0 \%$ ) compared with gemcitabine alone [5]. The higher peripheral neuropathy rate associated with FOLFIRINOX is secondary to the systemic delivery of oxaliplatin, a platinum-based cytotoxic drug which causes peripheral nerve damage [6].

Given these toxicities, alternative regional delivery methods have been developed to limit exposure of drugs to the systemic circulation and enhance tumor uptake and accumulation. Several studies over the past few decades have compared selective arterial delivery to intravenous delivery in PDAC and demonstrated improved response rates to a variety of agents [7]. This technique has evolved over the years, and the armamentarium of regional arterial delivery includes celiac axis infusion alone or in combination with selective embolization and isolated hypoxic perfusion [8]. Beyond PDAC, regional hepatic arterial delivery of cytotoxic therapy has been is relatively widespread use for colorectal liver metastases [9], and a recent clinical trial demonstrated that Pressure Enabled Drug Delivery (PEDD) of chimeric antigen receptor T-cells (CAR-T) via the hepatic artery resulted in promising treatment responses 
in liver metastases resistant to systemic treatment [10]. Further, the combination of pressurized arterial delivery of CAR-T and internal radiation achieved tumor growth reduction in 4 out of 6 patients, with 5 out of 6 showing reduced expression of serum markers such as CA-19-9 and minimal side-effects [11]. In a patient with liver metastases secondary to PDAC, three doses of anti-CEA CAR-T were regionally delivered via hepatic artery infusion (HAl) using PEDD technology; durable and sustained metabolic responses were observed in the liver for 13 months [10]. The PEDD method has also been evaluated for delivery of anti-PD-1 checkpoint blockade to colorectal liver metastases in a preclinical murine portal vein delivery model with demonstration of enhanced tumor control compared to systemic delivery [12].

In a recent study using a syngeneic orthotopic PDAC mouse model, the previously described PEDD method was modified to deliver gemcitabine by Pancreatic Retrograde Venous Infusion (PRVI) to the pancreatic tumor [13]. In this model, retrograde venous infusion through the portal vein with pancreatic venous isolation allowed for gemcitabine delivery to PDAC tumors at pressures $20-30 \mathrm{mmHg}$ above baseline intra-tumoral pressures and resulted in significantly higher tumor uptake of the cytotoxic drug as well as improved tumor response rates. Based on this regional delivery literature, we hypothesize that PRVI of oxaliplatin in an orthotopic mouse model of PDAC would improve tumor response rates while limiting the neurotoxic side effects of the drug.

\section{Materials And Methods}

\section{Cell Culture}

The murine PDAC cell line KPC4580P, a gift from Dr. Jen Jen Yeh of University of North Carolina, was established from male LSL-KrasG12D/+; LSL-Trp53R172H/+; Pdx1Cre/+; LSL-Rosa26 Luc/+ mouse as previously described [14]. The cells were cultured with DMEM : F12 media in 50:50 ratio with $10 \%$ FBS and $1 \%$ penicillin-streptomycin antibiotics at $37^{\circ} \mathrm{C}$ with $5 \% \mathrm{CO}_{2}$. Cells were used within three passages of thawing between experiments.

\section{Animal Experiments}

All animal experiments were approved by the Institutional Animal Care and Use Committee (IACUC) of University of California, San Diego (UCSD). Wild type (WT) 12-14 week old adult male C57BL/ 6 (Jackson Laboratories, Bar Harbor, ME) were utilized in an orthotopic pancreatic tumor model as previously described [15]. Briefly, 500,000 KPC-4580P cells were injected SQ and allowed to grow for 10 days. The tumors were harvested, and small pieces $\left(\sim 2 \mathrm{~mm}^{3}\right)$ were implanted onto the tail of the mouse pancreas. Once the tumors reached $5 \mathrm{~mm}$ diameter, the mice underwent PRVI as previously described [13]. In short, PRVI oxaliplatin and PRVI saline mice underwent laparotomy followed by temporary pancreatic venous isolation with microvascular clamps (Roboz Surgical, Boston, MA), and portal vein cannulation. Mice were randomized to receive either oxaliplatin $2 \mathrm{mg} / \mathrm{kg}$ in $100 \mu \mathrm{L}$ saline or saline $(100 \mu \mathrm{L})$, infused at a rate of $5 \mathrm{~mL}$ per minute. The venous isolation was released after $5 \mathrm{~min}$ of clamp time. The portal vein 
puncture site was covered with Surgicel (Ethicon, Raritan, $N J$ ). Intraperitoneal injections of oxaliplatin at concentrations of $2 \mathrm{mg} / \mathrm{kg}, 20 \mathrm{mg} / \mathrm{kg}$, or $40 \mathrm{mg} / \mathrm{kg}$ were used as systemic controls. Mice $(\mathrm{n}=3)$ from the PRVI oxaliplatin and systemic oxaliplatin groups were randomly selected for euthanasia at $3 \mathrm{~h}$ postinfusion to evaluate platinum concentrations within tumors as well as in plasma. All mice were euthanized on day 7 , and tumors and foot skin (peripheral nerves) were harvested and evaluated for treatment response.

\section{Histopathological Analysis}

Tissue (tumor and foot skin) specimens collected from euthanized mice 7 days after infusion underwent histologic evaluation. Slides containing serial tissue sections of paraffin-embedded tissue blocks were deparaffinized with xylene and hydrated using ethanol gradient. For IHC staining using the neuronal marker PGP 9.5, slides were deparaffinized with xylene, hydrated using ethanol gradient, and quenched with methanol/hydrogen peroxide, followed by antigen retrieval with citrate buffer $(\mathrm{pH}=6)$. Tissue sections were blocked with horse serum and incubated with primary antibody anti-mouse PGP 9.5 (ProteinTech) followed by HRP-conjugated secondary antibody. DAB (3,3'-diaminobenzidine) solution was used to develop the staining and counterstained with hematoxylin. The slides were imaged using an Olympus SC100 microscope at $20 \times$ magnification. The number of PGP 9.5 stained foci were counted independently within the epithelium and the dermal layers as Intra-Epidermal Nerve Fibers (IENF) and Sub-epidermal Neural Plexi (SNP), respectively (19) in 5 mice/group with each data point representing the mean value from 3 regions of interest per mouse.

\section{Measurement Of Platinum Concentration}

Tumor tissue from animals was collected into $15 \mathrm{~mL}$ conical tubes, documenting actual tissue weights, and washed with $70 \%$ ethanol. In the fume hood, $571 \mu \mathrm{L}$ of Metal Free Nitric Acid (37\%) was added to the tube containing the tissue and vortexed at high speed for 10 seconds. Samples were left in a fume hood overnight for tissue digestion. Once tissue was fully dissolved, the total weight of the mixture was determined and increased to a final volume of $10 \mathrm{~mL}$ with ultrapure distilled water. Samples were centrifuged and the supernatant was filtered and collected for Inductively Coupled Plasma- Mass Spectroscopy analysis. The ICP-MS analysis was done on a Thermo Scientific iCAP RQ ICP-MS in the Environmental and Complex Analysis Laboratory on the UC San Diego campus. The analysis was conducted in standard (STD) mode monitoring 159Tb and 209Bi as internal standards. The results were reported as ppb of platinum relative to the initial tumor weight [16].

\section{Motor Nerve Conduction Velocity}

Mice were anesthetized using 2.5 to $4 \mathrm{ppm}$ isoflurane in oxygen and transferred to a circulating waterheated pad, with anesthesia maintained via a face mask connected to the isoflurane regulator. Two 
recording electrodes (platinum-tipped sub-dermal needle electrodes, Grass Technologies) were inserted into the interosseous muscles between the animal's second, third, and fourth toes, and secured to the heating pad with lab tape. A grounding electrode was placed into the skin at the neck. A PowerLab stimulator (AD instruments) was set to deliver a $200-\mathrm{mV}, 50-\mu$ sec-duration square-wave stimulus every 2 sec. The stimulating electrode was inserted into the ankle near the Achilles tendon and adjusted until the resulting $M$ waves were clear and maximal. The electrode was removed and inserted into the sciatic notch at the hip and resulting $M$ waves were recorded, as previously described [17]. The distance between the stimulation sites at the hip and ankle was measured and divided by the time (latency) between the peaks of proximal and distal sites to obtain the motor nerve conduction velocity (MNCV).

\section{Statistical Analysis And Data Availability}

All data were analyzed using GraphPad Prism 8.0 software (GraphPad Software Inc, La Jolla, CA). Student's $t$ test with 2-tailed hypothesis was used to compare 2 groups or 1-way analysis of variance with Tukey's multiple comparison test was used for multiple groups. Survival was compared between groups by Kaplan-Meier analysis. The datasets generated during and/or analyzed during the current study are available from the corresponding author on reasonable request.

\section{Results}

\section{Induction of peripheral neuropathy in mice after acute systemic oxaliplatin exposure}

Peripheral neuropathy is a known side effect of chronic exposure to oxaliplatin over several weeks to months [18]. However, to establish an acute model of peripheral neuropathy in mice, we exposed KPC4580P orthotopic pancreatic tumor-bearing C57BL/6 mice to escalating single doses of oxaliplatin to observe changes in peripheral nerves within a seven-day period. The dose of $40 \mathrm{mg} / \mathrm{kg}$ was highly toxic to the mice resulting in greater than $90 \%$ death within five days of injection (Figure $1 \mathrm{~A}$ ). Doses of 20 $\mathrm{mg} / \mathrm{kg}$ and lower were well tolerated by the mice without signs of systemic toxicity. To further confirm the peripheral neuropathic effects of systemic oxaliplatin, motor nerve conduction velocity (NCV) measurements were performed (Figure 1B). Compared to the historical average of C57BL/6 mice of this age [19] (red dotted line with 95\% confidence interval in blue, Figure 1C), the average MNCV of both the 20 and $2 \mathrm{mg} / \mathrm{kg}$ groups were lower with the $20 \mathrm{mg} / \mathrm{kg}$ dose group showing a significant $(P<0.01)$ slowing compared to the $2 \mathrm{mg} / \mathrm{kg}$ dose group. These experiments confirm the potential for oxaliplatin to generate large motor fiber damage within 7 days of exposure.

Pancreatic Retrograde Portal Venous Infusion (PRVI) reduced tumor burden at a lower oxaliplatin dose.

To localize oxaliplatin exposure to tumors, we employed PRVI to deliver oxaliplatin retrograde through the portal vein to the pancreas. Examination of tumor burden 7 days after procedure (Figure 2A) shows that 
the average tumor weight of the PRVI group $(0.66 \pm 0.07 \mathrm{~g})$ at a concentration of $2 \mathrm{mg} / \mathrm{kg}$ was significantly lower than the PRVI saline group $(1.09 \pm 0.09 \mathrm{~g}, \mathrm{p}<0.05)$ and also lower than the group of mice exposed to the same low concentration of oxaliplatin through systemic delivery $(1.07 \pm 0.1 \mathrm{~g}, \mathrm{p}<$ 0.05). The average tumor burden at the higher concentration of $20 \mathrm{mg} / \mathrm{kg}$ through systemic delivery $(0.66$ $\pm 0.15 \mathrm{~g})$ was not statistically different than the PRVI delivery of oxaliplatin at $2 \mathrm{mg} / \mathrm{kg}(\mathrm{p}=0.99)$. Exploratory analysis of platinum accumulation within a subset of tumors at $3 \mathrm{~h}$ post infusion showed that the PRVI oxaliplatin $2 \mathrm{mg} / \mathrm{kg}$ mice had a 4 -fold increased tumor accumulation of platinum compared to systemic delivery at the same concentration. By comparison, a 10-fold increase in the systemic dose from $2 \mathrm{mg} / \mathrm{kg}$ to $20 \mathrm{mg} / \mathrm{kg}$ of oxaliplatin generated only a 2-fold increase in tumor platinum concentration (Figure 2B). Of note, these differences did not reach statistical significance due to the small sample sizes. The plasma concentration of platinum, however, was not affected by the mode of delivery, with values proportional of the initial concentration of the drug (Figure 2C). This finding indicates that $\mathrm{PRVI}$ is not preventing systemic exposure to drug.

\section{Therapeutic systemic doses of oxaliplatin generated peripheral nerve damage.}

Quantification of PGP 9.5 positive nerve fibers, a pan-neuronal cytoplasmic marker, allows assessment of nerve fiber density in the foot skin of mice treated with oxaliplatin (Figure $3 \mathrm{~A}$ ). Even the low concentration of $2 \mathrm{mg} / \mathrm{kg}$ significantly ( $\mathrm{p}<0.05$ ) reduced the density of intra-epidermal nerve fibers (IENF) compared to the saline infusion with no significant difference between the PRVI and systemic infusion groups (Figure 3B). There were no significant differences in sub-epidermal neural plexi (SNP) between either of the 2 $\mathrm{mg} / \mathrm{kg}$ groups and the saline infusion group. However, the nerve damage seemed to be a dose-dependent phenomenon, and both the IENFs and the SNPs were reduced in the group that received $20 \mathrm{mg} / \mathrm{kg}$ compared to the other groups (Figure 3C), showing the detrimental effects of the dose of oxaliplatin necessary to affect tumor growth by systemic delivery.

\section{Discussion}

Limiting systemic exposure of cytotoxic drugs while maintaining effectiveness is the key to avoiding undesirable side effects. High-dose $(40 \mathrm{mg} / \mathrm{kg})$ oxaliplatin can be extremely toxic and lethal in mice, and only one study has reported peripheral neuropathy at this dose in a survival mouse model [20]. While clinically relevant therapeutic regimens of systemic oxaliplatin at $2-4 \mathrm{mg} / \mathrm{kg}$ every 2 days over 3 weeks can reliably reach cumulative $40 \mathrm{mg} / \mathrm{kg}$ with associated peripheral neuropathy [18], our model was not compatible with repeated dosing, since performing multiple survival PRVI surgeries on mice is not technically and ethically feasible.

Although single-dose oxaliplatin is not expected to generate a durable response in PDAC, the pathophysiological effects of chemotherapy-induced peripheral neuropathy exhibited by our mouse model are representative of the human disease and thus serve as an appropriate model to compare delivery systems. Remarkably, PRVI oxaliplatin delivery was able to reduce PDAC tumor burden at a concentration ten-fold lower than what was required through systemic delivery. We believe this is due to 
the ability of PRVI to achieve higher tumor accumulation of the cytotoxic agent compared to the nonpressurized systemic delivery system. In a human subject, where repeated dosing of oxaliplatin through PRVI is possible, this close to 2 -fold reduction in tumor growth compared to control mice can amount to a significant therapeutic benefit to patients who are not candidates for surgical tumor resection.

Immunohistochemical analyses of peripheral nerves in our study showed that peripheral neuropathy is a consequence of total drug exposure. PRVI in our mouse model was not able to significantly reduce the systemic exposure of oxaliplatin, based on the similar plasma platinum levels between systemic and PRVI delivery of the same dose. However, the higher tumor accumulation of the drug with PRVI allows use of a much lower dose of drug than needed with systemic delivery. These findings are similar to our earlier observations with gemcitabine delivery using PRVI, where a 7-fold increased tumor accumulation of the drug was achieved [13], but the fraction of total drug absorbed by the tumor was still very low as suggested by similar systemic drug exposure. Tumor burden was reduced with a single dose of oxaliplatin at $2 \mathrm{mg} / \mathrm{kg}$ via PRVI, but we envision that PRVI can potentially be used to deliver multi-drug regimens or in combination with systemic delivery of less toxic, synergistic agents. An FDA-approved delivery device (TriSalus Infusion System, TIS-21120-60, TriSalus Life Sciences, Westminster CO) is currently in use for hepatic arterial delivery and could also be used to accomplish PRVI clinically. For PRVI, the device would be introduced into the portal venous system by percutaneous transhepatic access though a technique similar to that used for portal vein embolization. Therefore, PRVI in humans could be performed much less invasively than in mice such that repeated dosing would be feasible, and a clinical trial evaluating this approach is currently in development.

\section{Statements And Declarations}

DJ, BC, and SK are a part of TriSalus ${ }^{\mathrm{TM}}$ Life Sciences, Inc., Westminster, CO, USA. RW received an unrestricted gift from TriSalus ${ }^{\mathrm{TM}}$ Life Sciences that was used to partly support this research. The other authors declare no competing financial interests.

\section{Acknowledgements}

We would like to acknowledge Prof. Nigel Calcutt, Department of Pathology, UCSD and Dr. Neal Arakawa, Environmental and Complex Analysis Laboratory (ECAL), UCSD for their inputs to this study. This study was partly funded by TriSalus ${ }^{\mathrm{TM}}$ Life Sciences, Inc., Westminster, CO, USA.

\section{References}

1. Siegel RL, et al., Cancer Statistics, 2021. CA: A Cancer Journal for Clinicians, 2021. 71(1): p. 7-33.

2. Tempero MA, et al. Pancreatic Adenocarcinoma, Version 2.2021, NCCN Clinical Practice Guidelines in Oncology. J Natl Compr Canc Netw. 2021;19(4):439-57.

3. Kang J, et al. Nab-paclitaxel plus gemcitabine versus FOLFIRINOX as the first-line chemotherapy for patients with metastatic pancreatic cancer: retrospective analysis. Investig New Drugs. 
2018;36(4):732-41.

4. Tong $\mathrm{H}$, et al. The benefits of modified FOLFIRINOX for advanced pancreatic cancer and its induced adverse events: a systematic review and meta-analysis. Sci Rep. 2018;8(1):8666.

5. Conroy T, et al. FOLFIRINOX versus gemcitabine for metastatic pancreatic cancer. N Engl J Med. 2011;364(19):1817-25.

6. Cersosimo RJ. Oxaliplatin-associated neuropathy: a review. Ann Pharmacother. 2005;39(1):128-35.

7. Liu F, et al. Regional intra-arterial vs. systemic chemotherapy for advanced pancreatic cancer: a systematic review and meta-analysis of randomized controlled trials. PLoS One. 2012;7(7):e40847.

8. Lorenz $\mathrm{M}$, et al. Regional chemotherapy in the treatment of advanced pancreatic cancer - is it relevant? Eur J Cancer. 2000;36(8):957-65.

9. Kemeny NE, et al. Conversion to resectability using hepatic artery infusion plus systemic chemotherapy for the treatment of unresectable liver metastases from colorectal carcinoma. $\mathrm{J}$ Clin Oncol. 2009;27(21):3465-71.

10. Katz SC, et al. HITM-SURE: Hepatic immunotherapy for metastases phase Ib anti-CEA CAR-T study utilizing pressure enabled drug delivery. Journal for ImmunoTherapy of Cancer. 2020;8(2):e001097.

11. Katz SC, et al. HITM-SIR: phase Ib trial of intraarterial chimeric antigen receptor T-cell therapy and selective internal radiation therapy for CEA+ liver metastases. Cancer Gene Ther. 2020;27(5):341-55.

12. Chai LF, et al., Regional pressure-enabled drug delivery of anti-PD-1 agent for colorectal liver metastases improves anti-tumor activity without increased hepatic toxicity. The Journal of Immunology, 2019. 202(1 Supplement): p. 71.1-71.1.

13. Shankara Narayanan JS, et al. Pressure-enabled delivery of gemcitabine in an orthotopic pancreatic cancer mouse model. Surgery. 2020;168(3):448-56.

14. Narayanan JSS, et al. Irreversible Electroporation Combined with Checkpoint Blockade and TLR7 Stimulation Induces Antitumor Immunity in a Murine Pancreatic Cancer Model. Cancer immunology research. 2019;7(10):1714-26.

15. Shankara Narayanan JS, et al., A Syngeneic Pancreatic Cancer Mouse Model to Study the Effects of Irreversible Electroporation. J Vis Exp, 2018(136).

16. Li J, et al. In vivo biodistribution of platinum-based drugs encapsulated into multi-walled carbon nanotubes. Nanomed Nanotechnol Biol Med. 2014;10(7):1465-75.

17. Jolivalt CG, et al. Peripheral Neuropathy in Mouse Models of Diabetes. Curr Protoc Mouse Biol. 2016;6(3):223-55.

18. Marmiroli P, et al. Susceptibility of different mouse strains to oxaliplatin peripheral neurotoxicity: Phenotypic and genotypic insights. PloS one. 2017;12(10):e0186250-0.

19. Walsh ME, et al. Use of Nerve Conduction Velocity to Assess Peripheral Nerve Health in Aging Mice. The Journals of Gerontology: Series A. 2014;70(11):1312-9.

20. Sprowl JA, et al., Oxaliplatin-induced neurotoxicity is dependent on the organic cation transporter OCT2. Proceedings of the National Academy of Sciences, 2013. 110(27): p. 11199-11204. 


\section{Figures}

\section{Figure 1}

Acute exposure of high dose systemic oxaliplatin can cause peripheral neuropathy in mice. A) Kaplan Meier survival analysis of C57BL/6 harboring orthotopic pancreatic tumors exposed to different systemic (IP) doses of oxaliplatin shows the dose of $40 \mathrm{mg} / \mathrm{Kg}$ causing acute toxicity and death in this model. B) Motor Nerve Conduction Velocity (MNCV) measurement shows a reduction of nerve function. Schematic of the MNCV experiment on mice showing the placement of recording electrode in the intraosseus muscles electrode (a), grounding electrode (b) and the stimulating electrodes at the ankle (c) and sciatic notch. Time taken for the nerve impulse to travel $\mathrm{c}$ to $\mathrm{d}$ was measured to calculate the MNCV. C) MNCV data represented as mean \pm SEM of 5 mice per group of systemic oxaliplatin delivery with each data point representing the mean value from 3 repeated measurements per mouse. Red dotted line represents the average MNCV of untreated mice with $95 \%$ confidence intervals (blue dotted lines) reported elsewhere [19].

\section{Figure 2}

Pancreatic retrograde portal venous infusion (PRVI) of oxaliplatin can minimize the neuropathic effects of systemic oxaliplatin delivery by reducing the dose required for effective tumor control. A) Tumor burden in the orthotopic PDAC mice 7 days post treatment shown as mean \pm SEM of tumor weights among the survivors of $n=15$ mice in each group. B) Platinum concentration in the tumor and the circulating platinum levels in the plasma (C) were measured $3 \mathrm{~h}$ post infusion using inductively coupled plasma mass spectrometry (ICP-MS) and represented as mean \pm SEM parts per billion in 3 mice per group.

\section{Figure 3}

Neuropathic effects of systemic vs PRVI oxaliplatin delivery. A) Immunohistochemical staining for the measurement of number of fibers that express PGP9.5 neuronal marker in foot skin 7 days post oxaliplatin infusion shows the extent of neurodegeneration. Scale bar $=20 \mu \mathrm{m}$. Black arrow indicates IENF and red indicates SNP. The density of (B) Intra-epidermal nerve fibers (IENF) and (C) Sub-epidermal Neuronal Plexi (SNP) were compared between the groups under a light microscope at 20X magnification. 
Data represented as mean \pm SEM of 5 mice/group with each data point representing the mean value from 3 regions of interest per mouse.

\section{Figure 4}

Schematic representation of the study design and outcome, created with BioRender.com. 